\title{
10. ERGEBNISSE UND AUSBLICK
}

Die Durchsicht der Forschungsliteratur zum mittelalterlichen Dialog ließ drei wichtige Desiderate auf diesem Gebiet erkennen. Die lateinische Dialogliteratur ist noch nicht vollständig erschlossen; vor allem im Spätmittelalter gibt es noch zahlreiche Texte, die unediert sind oder nur in schwer zugänglichen Ausgaben vorliegen. Es fehlt außerdem ein angemessener Dialogbegriff für die mittellateinische Literatur: Früher wurden mittelalterliche Dialoge nach den Maßstäben der antiken und frühneuzeitlichen Dialoge gemessen, später setzte sich zwar die Erkenntnis durch, dass historisch adäquate Beschreibungskriterien notwendig seien, aber die Diskussion wurde von neuen Verständnisformen von Dialog als mündlichem Gespräch, als gelungener Kommunikation oder als unterschiedlich definierter Dialogizität dominiert. Das dritte Desiderat der Forschung ist eine Typisierung mittelalterlicher Dialogschriften auf quantitativ ausreichender Basis.

Um diese Desiderate zu erfüllen, wurde folgendermaßen vorgegangen. Zuerst mussten Kriterien gefunden werden, um ein brauchbares Korpus von Texten abzugrenzen. Dieses Korpus wurde durch ein ausführliches Repertorium erschlossen, das den zweiten Teil dieser Untersuchung bildet. Aufgrund der empirischen Erfassung der Texte wurden sechs verschiedene Typen beschrieben, sowie einige Schriften, die sich kaum einordnen lassen. Diese Beschreibung bildete die Grundlage, um einen Dialogbegriff zu erarbeiten und die Frage zu beantworten, ob es in der mittellateinischen Literatur eine Textsorte Dialog gibt oder vielmehr verschiedene dialogische Textsorten auszumachen sind.

An dieser Stelle werden nun die einzelnen Schritte der Forschung zusammengefasst (10.1). Bei der Beschreibung der Typen wurde die Notwendigkeit ersichtlich, frühere Texte zu berücksichtigen, die den diachronischen Hintergrund erhellen und somit eine genauere Bestimmung und Eingrenzung der Typen erlauben. Die Beobachtungen in Bezug auf die früh- und hochmittelalterliche Dialogliteratur finden sich in den Typenbeschreibungen integriert, mir schien aber wichtig, die Erkenntnisse, die sich daraus ableiten lassen, zusammenzufassen, was unter 10.2. „Diachronische Veränderungen“ geschieht. Einige Beobachtungen zur Beziehung zwischen Dialogform und Mündlichkeit finden sich schließlich im Anhang.

(C) Carmen Cardelle de Hartmann, 2007 | DOI:10.1163/9789047420637_012

This is an open access chapter distributed under the terms of the CG BY-NC 4.0 license. 


\subsection{Zusammenfassung}

Am Beginn dieser Arbeit wurde die Frage gestellt, was mittelalterliche Autoren und Rezipienten als Dialog bezeichneten. Die Aussagen über den Dialog in metaliterarischen Texten (Enzyklopädien, Accessus, Kommentaren) sind nicht eindeutig und beziehen sich teilweise nur auf die kommentierten Werke. Trotzdem lassen sich einige mittelalterliche Ansichten und Auffassungen ausmachen. An den Dialogen wurde das didaktische Potenzial und die Attraktivität für einfache Leser geschätzt. Es wurde auch beobachtet, dass sie den Leser emotional zu bewegen vermögen und dass die Person des Sprechers den Aussagen eine größere Autorität verleihen kann. Die Autoren schlagen sich mit einigen Widersprüchen herum: Dialogus wird häufig als Zwiegespräch gedeutet, obwohl einige dialogi mehr als zwei Figuren auftreten lassen; der Dialog wird der Personensprache zugeordnet, aber gleichzeitig wird beobachtet, dass Abschnitte in der Autorsprache durchaus dazu gehören können. Die Verfasser von Dialogen beschreiben ihr Vorgehen als „durch Fragen und Antworten“ und beziehen sich auf den Dialog vor allem als Dialogform (modus oder forma dialogi). Es wird angemerkt, dass diese Form das mündliche Gespräch evoziert, doch ist die weiter gehende Behauptung selten, zurückliegende tatsächlich geführte Gespräche wiederzugeben. Die Titelgebung in den mittelalterlichen Handschriften zeigt, dass Schriften mit dem Titel Dialogus in Personensprache, manchmal mit strukturell untergeordneten Abschnitten in Autorsprache, gestaltet sind. Sie lassen Figuren auftreten und sind mit nur wenigen Ausnahmen in Prosa geschrieben. Diatribische Texte und Ansprachen, die in der modernen Forschungsliteratur mitunter als dialogisch angesehen werden, werden im Mittelalter nicht als Dialogus bezeichnet.

Aus diesen Beobachtungen wurden zur Abgrenzung des Korpus einige wenige, klar erkennbare Merkmale herausgefiltert: Prosa und dominierende Personensprache mit direkt oder indirekt genannten Figuren. So entstand ein umfangreiches Korpus, das die Grundlage für die Typisierung und dadurch für die nähere Bestimmung des Dialogbegriffes bildete. Als zeitliche Grenzen wurden 1200 und 1400 gewählt, da die mittellateinische Textproduktion dieser Zeit noch wenig bekannt ist und der Erschließung bedarf. Alle Fragen, die Entstehung und Überlieferung der Texte betrafen, wurden in dem ausführlichen Repertorium diskutiert. In ihm finden sich auch detaillierte Inhaltszusammenfassungen der besprochenen Werke. 
Die Beschreibung der im Zeitraum 1200-1400 entstandenen Schriften wurde durch die Berücksichtigung der früheren Entwicklung und anderer, ähnlich gearteter Texte (zum Beispiel in Versform oder mit großen Anteilen an Autorsprache) ergänzt. Die mittelalterlichen Verfasser kombinieren gern Gestaltungsmerkmale aus verschiedenen Werken und Traditionen, wodurch unklassifizierbare Einzelwerke sowie eine Vielzahl von Übergangs- und Mischformen entstehen. Sie sind für die Beschreibung der mittellateinischen Literatur so wichtig wie die Werke, die sich in eine einzige Tradition oder Textsorte ohne Einschränkung einreihen lassen, und wurden deshalb berücksichtigt.

Im Korpus der dialogartigen Schriften sind vier große Typen zu erkennen, die stark vertreten sind und sich in Untertypen gliedern lassen; hinzu kommen zwei kleinere Textgruppen:

Lehrdialoge: Das prägende Merkmal der Lehrdialoge ist die LehrerSchüler-Beziehung zwischen den Dialogpartnern, die der Wissensvermittlung und der Unterweisung dient. Ihre einfachste Form, der Frage-Antwort-Dialog, geht auf die Antike zurück und ist mit Textsorten der Schulliteratur eng verbunden: die Erotapokriseis oder Quaestiones et responsiones, die Schulbücher in Frage-Antwort-Form und die Colloquia, einfache Texte in Personensprache zur Erlernung einer Fremdsprache. Die einfachsten Dialoge sind nur durch die Zuweisung der Fragen und Antworten an Figuren von den Quaestiones et responsiones oder den Schulbüchern zu unterscheiden. Die meisten Texte bieten jedoch eine kleine Ausgestaltung der Gesprächssituation, die darin besteht, dass die Figuren sich gegenseitig ansprechen, loben, ermutigen oder um Unterstützung bitten. Ein narrativer Rahmen sowie eine örtliche und zeitliche Situierung sind hingegen selten. Im Früh- und Hochmittelalter entstanden die Frage-Antwort-Dialoge im Umkreis von Schulen, im Hochmittelalter gelegentlich auch an weltlichen Höfen. Im Spätmittelalter werden sie an den Universitäten von anderen, den Lehrbedürfnissen besser angepassten Textsorten verdrängt. Bei der Durchsicht des diachronischen Hintergrunds wurde auch kurz auf Texte eingegangen, die aus Auflistungen von Wissens- und Rätselfragen bestehen. Sie wurden als Quellen für Witzdialoge mit zum Teil parodistischem Charakter verwendet, die im Frühmittelalter entstanden und sich im Spätmittelalter noch großer Beliebtheit erfreuten. Im Hochmittelalter kam ein kleiner Untertyp hinzu, der zwar durch die lateinischen Lehrdialoge beeinflusst sein mag, aber in erster Linie auf eine griechisch-arabische Dialogtradition zurückgeht: die alchemistischen Lehrdialoge. Sie sind durch den Esoterismus des vermittelten Wissens 
und den Initiationscharakter der Unterweisungssituation geprägt. Die monastischen Lehrdialoge zeigen eine besondere Einheitlichkeit durch die Orientierung nach einem prägenden Vorbild, den Dialogi Gregors des Großen, und wegen der Verbundenheit mit einem monastischen Publikum und seinen Interessen. Trotzdem sind sie bisher nicht als einheitliche Gruppe erkannt worden, vielmehr gibt es in der Forschung verschiedene Bezeichnungen und Abgrenzugen, die in Kapitel 2 diskutiert wurden. Dies führte zu einer Erörterung des Dialogs in der monastischen Literatur. Neben den monastischen Lehrdialogen sind auch monastische Streitgespräche zu erkennen, die im 12. Jahrhundert die Auseinandersetzungen unter den religiösen Orden und zwischen Kanonikern und Mönchen thematisieren und zum Typ der Streitgespräche gehören. Im Früh- und Hochmittelalter entstanden außerdem einige Werke, die sich den Dialog des Sulpicius Severus zum Vorbild nehmen. Sie inszenieren eine Gesprächsrunde, die die Leistungen einer Person diskutiert und damit einen Meinungsaustausch über unterschiedliche Themen verknüpft. Wegen ihres Inhalts werden sie biographische Dialoge genannt. Obwohl sie keine Lehrdialoge sind und große Ähnlichkeit mit den moralischen Gesprächen zeigen, wurden sie an dieser Stelle besprochen, um sie von jenen monastischen Lehrdialogen, die Erzählungen miteinschließen, abzugrenzen. Das wichtigste Unterscheidungsmerkmal ist das Verhältnis zwischen Reflexion und Erzählung: Im biographischen Dialog bildet die Erzählung den Leitfaden, aus dem sich Diskussionen über andere Themen entwickeln können; im monastischen Lehrdialog sind die Erzählungen hingegen illustrierende Beispiele für die dargelegte Doktrin. Der monastische Lehrdialog zeigt im Spätmittelalter einen Wandel, indem er neue Themen aufnimmt und sich auch an ein Publikum außerhalb des Klosters richtet. Ein weiterer Untertyp der Lehrdialoge, die moralischen Lehrdialoge, scheint nur aus heutiger Sicht als Gruppe definierbar und könnte ein Beispiel dafür sein, wie der monastische Lehrdialog den Bedürfnissen der Laien angepasst wird. In diesen Werken stehen die Figuren nämlich in einer ähnlichen Beziehung zueinander wie die Figuren der monastischen Lehrdialoge, eine leitet die andere als ihr geistiger Führer oder Ratgeber. Es handelt sich jedoch nicht mehr um Mönche, sondern um einen geistlichen Lehrer und einen Laien oder gar um zwei Laien. Die Themen beziehen auch Aspekte ein, die nur für Laien relevant sein können, wie zum Beispiel den Umgang mit politischem Misserfolg.

Streitgespräche: Die Streitgespräche sind im Spätmittelalter äußerst zahlreich. Sie inszenieren zwei konträre Positionen, die von zwei 
verschiedenen Figuren vertreten werden. Eine Position wird als wahr oder überlegen dargestellt, daher wird keine Annäherung angestrebt und keine auf eine Kompromisslösung ausgerichtete Argumentation entfaltet. Im Unterschied zu der thematischen Vielfalt der Streitgedichte beziehen sich die Streitgespräche nur auf Inhalte mit gesellschaftlicher Resonanz. Sie lassen sich thematisch in zwei Gruppen einteilen: die Streitgespräche gegen Juden und die Streitgespräche innerhalb des Christentums.

Streitgespräche gegen Fuden wurden bereits in der Spätantike und in Byzanz geschrieben, es ist jedoch fraglich, ob die mittellateinischen Werke nur auf diese Tradition zurückzuführen sind. Im Frühmittelalter entstehen zwei Texte, die sich nach einem spätantiken Streitgespräch zwischen Synagoga und Ecclesia zu orientieren scheinen, aber die Mehrzahl der im Hochmittelalter abgefassten Streitgespräche richten sich wahrscheinlich nicht gegen die Juden, wie sie vorgeben, sondern reagieren auf Entwicklungen innerhalb des Christentums. In ihnen findet sich kaum Polemik gegen das Judentum, sondern vor allem eine Verteidigung des christlichen Glaubens und eine Erläuterung seiner Grundlagen. Die Figur des Juden bleibt in der Regel sehr diffus. Seine Rolle besteht bloß darin, Einwände vorzubringen, und nicht, eine eigene Position vorzutragen und zu vertreten. In seiner Charakterisierung verraten die Autoren (mit der Ausnahme von Petrus Alfonsi) ihre Unkenntnis des zeitgenössischen Judentums. Die Werke richten sich an die Christen und sind wohl im Zusammenhang des Bildungsbestrebens und -aufschwungs in Folge der gregorianischen Reform zu sehen. Etliche Verfasser behaupten auch explizit, mit ihrem Werk ihre Glaubensgenossen bilden und informieren zu wollen. Die Anregung, diese Unterweisung als Streitgespräch mit einem Juden einzukleiden, dürfte einerseits von der Gegenwart jüdischer Bevölkerung in Mittelund Nordeuropa herrühren. Es scheint immer wieder spontane Diskussionen zwischen Christen und Juden gegeben zu haben; manche Autoren behaupten sogar, mit ihrem Werk eine solche wiederzugeben. Andererseits stehen diese Streitgespräche in Beziehung zu dem neuen Ansatz in der Theologie, mehr auf die Vernunft als auf die Autorität zu setzen. Die Diskussion mit einem Nichtchristen bot den passenden Rahmen, den Einsatz von Vernunftgründen in der Erklärung der Glaubenswahrheiten vorzuführen. Im Spätmittelalter ändert sich dieser Typ auffällig. Nach dem hohen Stand der hochmittelalterlichen Werke, deren Autoren unter den herausragenden Philosophen und Theologen ihrer Zeit zu finden sind, erscheinen nun lediglich Texte, die auf einem 
einfachen Niveau die Grundsätze des Glaubens behandeln und dies mit Kritik gegen die Juden in einem zum Teil scharfen Ton kombinieren. Die Verfasser sind meistens unbekannt oder unbedeutend, das anvisierte Publikum dürfte aus Klerikern oder auch Laien mit nur geringer theologischer Bildung bestehen.

Im Früh- und Hochmittelalter entstanden wenige Streitgespräche innerhalb des Christentums. Sie kamen punktuell in Zusammenhang mit umstrittenen Papstwahlen, vor allem aber als monastische Streitgespräche vor. Im Spätmittelalter hingegen haben sie geradezu Hochkonjunktur, was durch die heftigen Kontroversen der Zeit, auf die die Werke Bezug nehmen, zu erklären ist: den Armutsstreit, den Streit um die Machtbefugnisse des Papstes und das Große Schisma. Die Universitätspolemiken finden nur dann ihren Niederschlag in Streitgesprächen, wenn sie den akademischen Raum verlassen, was zum Beispiel bei Wyclif und seinen Anhängern geschieht. Alle Streitgespräche führen konträre Positionen vor, wobei in jedem Fall klar ist, welche Position der Autor unterstützt. Der Raum, den er der gegnerischen Meinung einräumt, schwankt sehr stark, was zur Differenzierung dieser Dialoge in drei Gruppen führt. Die erste Gruppe sind die Streitgespräche zwischen Lehrer und Schüler. In ihnen trägt ein Schüler die gegnerischen Ansichten vor. So kann der Verfasser in einem einzelnen Werk Argumente erörtern, die von unterschiedlichen Personen oder Gruppen vertreten werden. Der Schüler zeigt sich aktiv und wird an der Argumentation beteiligt, was diese Gruppe den philosophischen Dialogen annähert, doch geht es hier vornehmlich darum, bestimmte Thesen auf ihre Richtigkeit hin zu überprüfen. In den asymmetrischen Streitgesprächen bringt der Opponent (die Figur, die die gegnerische Seite vertritt) lediglich Einwände und Fragen vor. Seine eigene Einstellung ist kaum zu erkennen. Die symmetrischen Streitgespräche hingegen gewähren beiden Positionen gleichen Raum. In ihrer einfachsten Form sind sie Doppeltraktate, die einen fremden Text zitieren und Absatz für Absatz widerlegen.

Innerhalb dieses Kapitels wurden die Streitgespräche des Raimundus Lullus separat behandelt, da sie eine neue Tradition entstehen lassen. Lullus ist ein sehr origineller Autor, der unterschiedliche literarische Traditionen frei kombiniert und dessen Werke sich deshalb schwer einordnen lassen. Sein Einsatz für Mission und Kreuzzug erklärt, dass er sich auch für Muslime und Tartaren interessiert, die in anderen Streitgesprächen nicht vorkommen. Ein weiteres Ziel des Lullus besteht darin, die Anwendbarkeit seiner Ars als Arbeitsmethode zu beweisen. Sie soll helfen, Andersgläubige zu bekehren und die Zerwürfnisse unter 
Christen beizulegen. Die Anhänger des Lullus verfassten Dialoge in mehreren Sprachen, die nach seinem Vorbild entstanden.

Selbstbetrachtende Dialoge: Die selbstbetrachtenden Dialoge haben drei spätantike Werke als Vorbilder: die Consolatio Philosophiae des Boethius, die Soliloquia Augustins und die Synonyma Isidors von Sevilla. Die Gemeinsamkeit zwischen diesen Werken besteht in der Inszenierung eines Selbstgesprächs als Dialog, in dem das Ich in zwei Figuren verdoppelt wird. Eine der Figuren hat eine leitende Rolle und führt ihren Gesprächspartner zu einer tieferen Erkenntnis seiner selbst und Gottes. Im Frühmittelalter wurden diese drei Werke zwar eifrig abgeschrieben und kommentiert, doch entstanden keine Dialoge nach ihrem Vorbild. Erst im Hochmittelalter werden selbstbetrachtende Dialoge geschrieben, darunter ein Werk mit einer breiten Rezeption: die Soliloquia Hugos von St. Victor. Die selbstbetrachtenden Dialoge zeigen Berührungen und Überschneidungen mit anderen Textsorten. Thematisch sind sie den Gebeten und Meditationen sehr nahe; der gemeinsame Bezug auf die boethianische Consolatio bewirkt die Annäherung an die philosophischallegorische Prosimetra. Im 12. Jahrhundert finden sich außerdem selbstbetrachtende Dialoge in Versform, im 13. und 14. Jahrhundert hingegen verschwinden selbstbetrachtende Dialoge in Vers- oder in prosimetrischer Form fast gänzlich. Im Spätmittelalter ist die Vielfalt der selbstbetrachtenden Dialoge auffällig. Einige Werke knüpfen an das Soliloquium Hugos von St. Victor an, daneben gibt es Neuerungen, die durch die Inszenierung mystischer Erfahrungen einerseits und den Rückgriff auf die Antike andererseits entstehen. In einigen Werken kommt die Gesprächskonstellation Mensch und Gott vor, wobei allerdings nur ein Autor, Heinrich Seuse, klar und eindringlich behauptet, damit eine reale Erfahrung wiederzugeben. Sein Horologium Sapientiae verläuft in entgegengesetzter Richtung zu den früheren selbstbetrachtenden Dialogen: Statt ein Individuum durch zwei Figuren darzustellen, verschmelzen bei Seuse gelegentlich die zwei Personen in eine einzige Stimme. Der Rückgriff auf die Antike besteht einerseits in der Einführung eines neuen Modells, der senecanischen Schrift De remediis fortuitorum, andererseits in einem erneuerten Zugriff auf die zwei ältesten Vorbilder, Boethius und Augustin, und durch sie auf ihre antiken Modelle, insbesondere Cicero. Dies führt in Petrarcas Secretum zu einer individualisierten Charakterisierung der Figuren, vor allem des Franciscus, wie sie in den selbstbetrachtenden Dialogen des Mittelalters nicht zu finden ist. Diese hatten den auftretenden Menschen alle Menschen repräsentieren, ihn alle Last und alle Sünde der Menschheit tragen und 
beklagen lassen. Im Secretum hingegen wird die Figur des Franciscus als Individuum mit spezifischen Problemen dargestellt.

Im Spätmittelalter gibt es auch eine Überschneidung mit anderen Textsorten: Mit den Marienklagen in zwei Dialogen, in denen Maria einem Menschen ihren Schmerz beim Leiden ihres Sohnes schildert, und mit moralphilosophischen Florilegien und Traktaten, in Werken, die charakteristische Figuren oder eine kleine Rahmenhandlung aus der Tradition der selbstbetrachtenden Dialoge übernehmen.

Philosophische Dialoge: Die philosophischen Dialoge sind aus moderner Sicht die literarischen Dialoge schlechthin, doch bilden sie im Mittelalter nur eine kleine Gruppe. Die Texte nehmen nicht auf ein oder mehrere Modellwerke Bezug und erhalten auch keine Kohäsion etwa durch ein gemeinsames Publikum, ähnliche Inhalte oder eine ähnliche Darstellung. Wenn es darum geht, die Figuren zu bestimmen oder eine Situation zu inszenieren, greifen die Verfasser nach den Mitteln der anderen Gruppen, woraus philosophische Dialoge im Gewand von Lehrdialogen, von Streitgesprächen oder von selbstbetrachtenden Dialogen entstehen. Dies macht die Abgrenzung der Gruppe schwierig. Wie Lehrdialoge und selbstbetrachtende Dialoge können philosophische Dialoge eine Unterweisungssituation inszenieren. Der Unterschied zu diesen zwei Gruppen besteht jedoch in der aktiveren Rolle des Schülers, der sich - mindestens gelegentlich - an der Argumentation beteiligt. Die Form der Argumentation trennt philosophische Dialoge von Streitgesprächen: In ersteren findet sich mehr oder weniger ausgeprägt eine emergente Argumentation, die zu Verschiebungen in der Diskussion und neuen Erkenntnissen führt, in den Streitgesprächen ist die Argumentation hingegen auf die bloße Überprüfung von Thesen ausgerichtet. Im Spätmittelalter sind zwei große Gruppen innerhalb der philosophischen Dialoge zu unterscheiden. Die dialektischen Dialoge behandeln klar abgegrenzte Fragestellungen und orientieren sich an der scholastischen Methode. Die moralischen Gespräche bevorzugen eine rhetorisch geprägte Argumentation auf der Grundlage von Zitaten und Exempeln und erörtern eine Vielzahl von Themen, meist moralischer Natur. Einige Werke der Gruppe integrieren diese unterschiedlichen Inhalte durch eine Erzählung, die als Leitfaden dient. Diesen Kunstgriff haben sie mit den biographischen Dialogen des Früh- und Hochmittelalters gemeinsam.

Streitgespräche über das Heilsgeschehen und Satansprozesse: Diese Gruppe versammelt einige Werke, die das grundlegende theologische Problem des Heilsgeschehens, nämlich die Notwendigkeit der Menschwerdung 
Gottes, von einem Vertreter des Himmels und einem Vertreter der Hölle diskutieren lassen. Einige sind als Streitgespräche ohne situativen Rahmen gestaltet, sie werden jedoch von uns nicht als Teil des Typs Streitgespräch diskutiert, da ihnen der Bezug auf eine aktuelle Polemik mit politischer und sozialer Resonanz fehlt. Ihr Thema, die auftretenden Personen und die juristische Argumentation stellen eine Verbindung zu den Satansprozessen. Diese ihrerseits präsentieren die gleich geartete theologische Diskussion im Rahmen einer Gerichtsverhandlung, in der über die Rechtmäßigkeit der Erlösung befunden wird. Die Satansprozesse verstärken die juristische Komponente der Argumentation, gleichzeitig ist die Personensprache nicht mehr die ausschließliche Form der Darstellung, die dialogischen Passagen alternieren vielmehr mit narrativen Abschnitten. Es treten neben den Streitenden auch andere Figuren auf: der Richter, der oder die Angeklagte, zusätzliche Anwälte sowie das Publikum.

Dramatische Allegorien: Die dramatischen Allegorien bilden eine kleine Gruppe von Texten, die wie stark erweiterte Parabeln oder wie Lesedramen wirken. Sie lassen eine Vielzahl von Figuren - häufig Personifikationen - auftreten und verwenden die Personensprache zur Charakterisierung der Personen und zum Vorantreiben der Handlung, weshalb sie größere Ähnlichkeit zum Drama als zu den Dialogen der vier großen Typen erkennen lassen.

Dialogform und dialogische Textsorten: Die Untersuchung und Beschreibung der im Arbeitskorpus enthaltenen Schriften zeigte einige Merkmale, die in den meisten Werken vorkommen: Inhaltsorientiertheit, mangelnde Charakterisierung der Gesprächsteilnehmer, gleichbleibende Beziehung zwischen den Figuren, reduzierte oder nicht vorhandene Rahmenhandlung, Multifunktionalität der Dialogform, Vorliebe für Zwiegespräche. Diese Merkmale definieren den prototypischen Dialog; konkrete Dialoge sind als solche dadurch zu erkennen, dass sie mit diesem Prototyp weitgehend übereinstimmen, auch wenn sie einzelne Merkmale nicht aufweisen. Diese Definition des Dialogs erlaubt es uns, aus dem Arbeitskorpus ein Ergebniskorpus abzugrenzen, zu dem lediglich die Texte gehören, die dieser Definition entsprechen. Diese Texte gliedern sich in die vier großen Typen Lehrdialoge, Streitgespräche, selbstbetrachtende und philosophische Dialoge mit ihren Untertypen. Dazu gehören auch die Streitgespräche zur Heilsgeschichte sowie einige Werke, die nichtdialogischen Textsorten zuzurechnen sind und in ihnen eine Ausnahmeerscheinung darstellen (Briefdialoge, dialogisierte Planctus). Zum Ergebniskorpus gehören Texte und Textgruppen nicht, die 
diese Merkmale nicht aufweisen: dramatische Allegorien, Satansprozesse, philosophisch-allegorische Prosimetra.

Die erwähnten Dialogmerkmale sind die gemeinsame Basis des resultierenden Ergebniskorpus. Die dort enthaltenen Schriften unterscheiden sich allerdings unter weiteren wichtigen Aspekten - in der Beziehung der Figuren, dem Bezug auf den historischen Kontext, der Orientierung an Modellwerken, dem anvisierten Publikum -, die die Gliederung in Typen und Untertypen begründen. Wegen dieser Vielfalt ist es nicht möglich, sie in eine einzige Textsorte Dialog zusammenzufassen. Der mittellateinische Dialog ist als mögliche Ausgestaltung der Personensprache eine Präsentationsform, die konstitutives Merkmal einer Textsorte sein kann. Dies ist der Fall bei Lehrdialogen, Streitgesprächen, selbstbetrachtenden und philosophischen Dialogen, die deshalb als ,dialogische Textsorten' bezeichnet werden können. Die Präsentationsform Dialog kann aber auch als optionales Merkmal in anderen Textsorten vorkommen, wie zum Beispiel in der Gruppe der Streitgespräche zur Heilsgeschichte und Satansprozesse. Schließlich kann sie in einzelnen Werken, die nichtdialogischen Textsorten zugehörig sind, eingesetzt werden. Ein Beispiel dafür sind die dialogisierten Planctus und die Briefdialoge.

\subsection{Diachronische Veränderungen}

Die christlichen Dialoge der Spätantike, vor allem diejenigen, die von Kirchenvätern geschrieben wurden, sind für mittelalterliche Autoren Referenzwerke mit besonderer Autorität. Doch gibt es keine einfache Kontinuität oder allmähliche Weiterentwicklung, vielmehr sind Weiterführungen, Brüche und Neuansätze nebeneinander zu beobachten.

Isidor von Sevilla behandelte den Dialog nicht zusammen mit der schulischen Kanonliteratur seiner Zeit, sondern mit den Gebrauchstexten, insbesondere Schulbüchern und Pastoralschriften. In diesem Zusammenhang kommen Dialoge auch im Frühmittelalter vor. In jener Epoche werden vor allem die Traditionen der didaktischen Dialoge der Spätantike und verwandter Textsorten weitergeführt. Die Dialogliteratur der Zeit ist an die Schule und an das Kloster - zwei ohnehin engverwandte Bereiche - gebunden. Eine einzige Ausnahme bildet der Hof Karls des Großen, an dem Lehrdialoge entstehen, die wohl zum Teil auch erwachsene Mitglieder des Hofes und den Kaiser selbst als Publikum ansprachen. Schulbücher in Frage-Antwort-Form, Frage- 
Antwort-Dialoge, Quaestiones et responsiones, Colloquia und Witzdialoge aus der Spätantike sind weiter im Umlauf und neue Werke entstehen nach ihrem Vorbild. Vielgelesene spätantike Vorbilder, Cassians Collationes und vor allem Gregors des Großen Dialogi, lassen die Tradition der monastischen Lehrdialoge entstehen. Zur monastischen Literatur gehören auch die biographischen Dialoge, die sich nach dem Vorbild des Sulpicius Severus orientieren und durch ihn einige Charakteristika der philosophischen Dialoge annehmen. Selbstbetrachtende Dialoge werden in dieser Zeit nicht geschrieben, obwohl die später maßgeblichen spätantiken Vorbilder gelesen und zum Teil sogar kommentiert werden. Auch einige wenige Streitgespräche werden verfasst, aber es ist schwer festzustellen, inwieweit sie früheren Modellwerken folgen. Für diese Form kann es verschiedene Anregungen gegeben haben: ältere Streitgespräche, Streitgedichte, rhetorische Schulübungen.

Im Hochmittelalter kommt es zu einer Reihe von Neuerungen. Die selbstbetrachtenden Dialoge der Spätantike - Augustins Soliloquia, Boethius' Consolatio, Isidors Synonyma - finden jetzt Nachahmer, die sich verschiedener Formen bedienen. Im Bereich der Lehrdialoge erscheint ein neues Publikum, als in Nordfrankreich und in England einige Werke am Hof geschrieben werden. Die adligen und königlichen Gönner treten nun in den Lehrdialogen als Lernende oder (in der neuen Form der moralischen Lehrdialoge) als Ratsuchende auf. Unter den Verfassern finden sich vermehrt Weltkleriker und sogar Laien wie Adelard von Bath. Während Colloquia und Witzdialoge nicht mehr geschrieben werden (erst in der Renaissance werden diese Textformen wieder aufgegriffen), kommt ein neuer Untertyp hinzu, der an eine fremdsprachige Tradition anknüpft: die alchemistischen Dialoge. Die auffälligsten Änderungen ergeben sich aber bei den philosophischen Dialogen und den Streitgesprächen. Der Siegeszug der Dialektik leitet eine neue Form philosophischen und theologischen Denkens ein, die ihren Niederschlag in einer reichen Dialogproduktion findet. Allerdings scheinen die Autoren die geeignete Form für philosophische Dialoge erst noch zu suchen: Anselm kleidet sein Werk in die Form des monastischen Lehrdialogs, Eberhard von Ypern kombiniert Elemente des selbstbetrachtenden Dialogs und der Komödie, Abaelard greift nach den Streitgesprächen mit den Juden. Das überraschende Aufblühen dieses letzten Typs dürfte auch in diesem Kontext zu erklären sein, denn das Gespräch mit einem Andersgläubigen gibt den Verfassern den geeigneten Kontrapunkt für eine Verteidigung des eigenen Glaubens mit Vernunftgründen. Unter den Streitgesprächen entsteht ein Untertyp, 
das monastische Streitgespräch, in dem die zeitgenössischen Polemiken zwischen Kanonikern und Mönchen und zwischen traditionellem und Reform-Mönchtum ihren Niederschlag finden.

Ab 1200 sind neue Veränderungen zu verzeichnen, die man nach ihren Ursachen bündeln kann. Die Universitäten als neue Kulturzentren verursachen, direkt oder indirekt, Veränderungen in der Literatur. Mit ihnen entsteht ein neues Publikum und es bilden sich neue Wege für die Zirkulation der Texte. Die innerchristlichen Streitdialoge, die Themen mit politischen Implikationen behandeln, scheinen vielfach unter Universitätsabsolventen ihre Leser und Abschreiber gefunden zu haben. Auch die Satansprozesse dürften im Umkreis der Hochschulen verfasst und gelesen worden sein. Nicht weniger wichtig ist die Entwicklung spezifisch universitärer Textsorten für den Unterricht, die die Frage-Antwort-Dialoge, die philosophischen Dialoge und wohl auch die Streitgespräche gegen die Juden verdrängen. Die Probleme, die im Hochmittelalter in diesen Textsorten erörtert wurden, werden jetzt in den neuen Formen der Quaestiones, der Summen und der universitären Traktate diskutiert. Sogar bei einem nichtuniversitären Themenbereich, der Alchemie, verdrängen Textformen, die den universitären Textsorten nachgebildet werden, die Lehrdialoge. Frühe Streitgespräche - wie der Dialog des Petrus Alfonsi oder die Disputatio des Georgius - wurden auch wegen ihrer Auskünfte über das Judentum, den Islam oder die Patarener abgeschrieben. Später finden sich solche Informationen vor allem in Handbüchern und Dossiers für Disputanten und Inquisitoren. Die Disputationen mit Juden werden streng reglementiert und in Protokollen festgehalten. Die Dialoge hingegen sind situations- sowie weitgehend kontextungebunden und scheinen ein immer breiteres Publikum anzusprechen. Frage-Antwort-Dialoge und Streitgespräche gegen die Juden richten sich an einfache Kleriker oder gebildete Laien; franziskanische Streitgespräche zirkulieren nicht nur in diesem Orden, sondern auch an den Höfen oder bei Weltklerikern; sogar die monastischen Lehrdialoge sprechen ein Laienpublikum an und nehmen die Laien mitunter als Gesprächspartner in den Text auf. Vor allem in Italien bilden Laien ein Literaturpublikum, das neue Impulse gibt. Die meisten Laien, die als Dialogautoren hervortreten, sind Italiener: Georgius, Inghetto Contardo, Albertino Mussato, Albertano da Brescia, Geri d'Arezzo, Petrarca, Lombardo della Seta, Giovanni Conversino. Unter den Bürgern der norditalienischen Städte entsteht die humanistische Bewegung, die literarische Formen und Literatursprache erneuert, indem sie neuen Vorbildern folgt oder bekannte Vorbilder neu liest. 
Unter den neuen Vorbildern sind neuentdeckte Schriften wie die Briefe Ciceros, früher wenig beachtete Werke wie die Tragödien Senecas, altbekannte Texte wie das senecanische De remediis fortuitorum. Aber auch Werke wie die Soliloquia Augustins oder die boethianische Consolatio, die bereits Vorbildcharakter hatten, erscheinen jetzt in Petrarcas Secretum in neuem Licht. Petrarca selbst wird schnell zum Vorbild für andere Autoren, zum Beispiel für Lombardo und für Mussato. Der Bereich, in dem der Kontrast zwischen humanistischen Texten und solchen, die der mittelalterlichen Tradition folgen, am klarsten zum Vorschein kommt, sind die philosophischen Dialoge. Sie spalten sich nach 1200 in scholastische Dialoge, die stark formalistisch abstrakte Probleme erörtern, und moralische Gespräche, vor allem von humanistischen Autoren geschrieben, die im Gesprächston moralische und alltägliche Probleme diskutieren. Doch die wohl auffälligste Innovation der Humanisten ist die Individualisierung der Figuren, vor allem derjenigen, die den Autor vertreten. Franciscus bei Petrarca, Johannes bei Conversino und Lombardus stellen nicht mehr - oder nicht nur - einen Typus dar, sondern auch die spezifischen Probleme, individuellen Ansichten und persönlichen Anliegen ihres Autors.

Schließlich ist zu fragen, ob und wie das Spätmittelalter zum viel diskutierten Aspekt der Dialogizität Neues beigetragen hat ${ }^{1}$. Eine stilistische Dialogizität, wie Bachtin sie im Roman beschreibt, ist im Dialog nicht gegeben, aber doch wohl eine inhaltliche, die sich in der Vielfalt der von den Figuren vertretenen Ansichten zeigt (was Hempfer ,Polyperspektivik nennt). Der erste Eindruck, den mittelalterliche Streitgespräche, die ja prinzipiell zwei Standpunkte kontrastieren sollten, erwecken, ist, dass die mittelalterliche Literatur diese Polyperspektivik noch nicht kennt ${ }^{2}$. Die meisten Streitgespräche lassen vor allem eine Figur zu Wort kommen, während ihr Opponent auf das Vorbringen kurzer Einwände reduziert wird. Doch zeichnen sich bereits im Hochmittelalter Ansätze ab, die gegnerische Meinung neben der eigenen in einem Dialog wiederzugeben, und zwar dann, wenn der Autor in einer innerchristlichen Diskussion persönlich beteiligt ist. Im Spätmittelalter kann man bei symmetrischen Streitgesprächen und bei den Doppeltraktaten das Nebeneinanderstellen der Meinungen beobachten - wobei allerdings weiterhin einer Position eindeutig der Vorzug gegeben wird. Drei Werke gehen, jedes auf seine

\footnotetext{
Siehe auch oben 14-17.

2 Dies ist die Ansicht, die Friedlein (,Geleit") vertritt.
} 
Weise, einen Schritt weiter. In der Epistola pacis Heinrichs von Langenstein argumentieren die zwei Figuren nicht nur jeweils zugunsten des Erst- und des Zweitgewählten bei der doppelten Papstwahl des Jahres 1378, sondern zeigen sich sogar in der Lage, in der Diskussion zu einer für beide vertretbaren Kompromisslösung zu gelangen. Ganz zugespitzt wird die Pluralität der Meinungen und die vielfachen Möglichkeiten über bestimmte Probleme zu argumentieren, in einem Werk, das die kontrastierenden Positionen paradoxerweise nicht auf verschiedene Figuren verteilt, nämlich dem Dialogus Wilhelms von Ockham. Auf der Dialogebene bleibt der Text traditionell: Der Schüler fragt, der Lehrer hält lange Vorträge. Aber inhaltlich findet sich hier Erstaunliches, denn die Argumente für und wider jeden besprochenen Punkt werden sorgfältig ausgebreitet und die Entscheidung dem Leser überlassen. Eine andere Art der Zuspitzung betreibt Petrarca im offenen Ende seines Secretum. Franciscus zeigt sich von Augustinus überzeugt, ist aber nicht in der Lage, seine Anweisungen in die Tat umzusetzen; Vernunft und Wille stimmen nicht überein.

Die auffälligsten Neuerungen der Zeit zwischen 1200 und 1400 im Bereich der Dialogliteratur lassen sich in diesen drei Aspekten beobachten: die aktive und wachsende Beteiligung der Laien als Autoren und Rezipienten, die Ansätze zur Darstellung verschiedener Meinungen und die Lockerung oder Auflösung der Bindung des Dialogs an ein bestimmtes Publikum oder bestimmte Gebrauchssituationen.

\subsection{Ausblick}

Ziel dieser Arbeit war die Erschließung und Typologisierung der mittellateinischen Dialoge zwischen 1200 und 1400 und die Erarbeitung einer Definition des Dialogs, die für die mittellateinische Literatur angemessen ist. Diese Ziele wurden erreicht und damit der Forschung eine „Landkarte“ zur Verfügung gestellt, die ihr auf dem weiteren Weg Orientierung bieten kann. In dieser Arbeit machten sich auch etliche blinde Flecken bemerkbar, die noch erschlossen werden müssen. Wiederholt mussten knappe Hinweise auf Textgruppen gegeben werden, die noch ungenügend bekannt sind, wie die Frage-Antwort-Dialoge und die Quaestiones et responsiones des Früh- und Hochmittelalters. Bei manchen von ihnen, wie zum Beispiel den Gebeten und Meditationen, fehlt noch eine literarhistorische Einordnung und Typisierung. Der Ansatz dieser Arbeit, der die Gemeinsamkeiten unter den Texten 
suchte, hat jedoch nicht verdeckt, dass für viele dieser Texte eine Einzelinterpretation notwendig wäre, die ihre komplexe Beziehung zur Tradition und ihre eigene Innovation herausarbeitet. Auch bei der Erschließungsarbeit wurden die Grenzen bemerkbar, die durch die noch unvollständige Katalogisierung der europäischen Handschriftenbestände bedingt sind; in diesen noch unbekannten Handschriften schlummern mit großer Wahrscheinlichkeit weitere Dialoge, die unser Bild ergänzen oder korrigieren könnten. Besonders relevant wäre die Untersuchung der Wechselwirkungen zwischen volkssprachlichen und lateinischen Literaturtraditionen. Dies wären weitere Schritte auf dem Weg zu einer Geschichte der mittelalterlichen Literatur, die lateinische wie volkssprachliche Traditionen gleichermaßen berücksichtigt und im kulturellen Geflecht ihrer Zeit verortet.

Faciendi plures libros nullus est finis... 